\title{
Entwicklung von energieautarken, intelligenten Ladehilfsmitteln am Beispiel des inBin
}

\author{
Development of Self-sustaining Intelligent Load Carriers Using the Example of \\ the inBin
}

Jan Sören Emmerich*

Moritz RoidI*

Tobias Bich**

Michael ten Hompel*

* Lehrstuhl für Förder- und Lagerwesen FLW, TU Dortmund

** Fraunhofer-Institut für Materialfluss und Logistik IML, Dortmund

D as autonome, intelligente Ladehilfsmittel verkörpert die Idee des Internets der Dinge in der Intralogistik in Reinform. Am Beispiel des inBin wird das Energy-Harvesting in der Intralogistik betrachtet und gezeigt, dass ein Behälter mit komplexen logistischen Funktionen unter realistischer Umgebungsbeleuchtung durch Solarzellen betrieben werden kann.

[Schlüsselwörter: Internet der Dinge, Intelligenter Behälter, Energy-Harvesting, Solarzellen, Intralogistik, Sensornetzwerk]

$\mathbf{T}$ he autonomous and intelligent load carrier embodies the idea of the Internet of things in intralogistics. Using the example of the inBin, the energy harvesting is examined in the intra-logistics and it is shown that photovoltaic cells can power a small container (bin) with complex logistical functions under realistic lighting conditions.

[Keywords: internet of things, intelligent bin, energy harvesting, photovoltaic cell, intra-logistics, sensor network]

\section{EINLEITUNG}

Flexibilität und Wandelbarkeit spielen in der Logistik eine immer wichtigere Rolle, da die Einsatzgebiete einer gestiegenen Komplexität und Dynamik ausgesetzt sind. Bei sich ändernden Umweltbedingungen steht dabei insbesondere eine einfache Erweiterbarkeit und Modifizierbarkeit bestehender und neuer Anlagen im Vordergrund. Eine erhöhte Wandelbarkeit von Materialflusssystemen kann über eine dezentrale Steuerung erreicht werden. Eine konsequente Modularisierung der Förder- und Steuerungstechnik macht so selbstorganisierte Materialflusssysteme realisierbar - das "Internet der Dinge in der Intralogistik" (vgl. hierzu z. B. [NH10; BH07; GCK08a; GCK08b; May09; HFR08]).

Ein besonderes Augenmerk verdient hierbei der Umstand, dass das Transportgut in der Regel die treibende
Kraft in der Steuerung sein soll, indem es die angebotenen Dienste entsprechend seiner Ziele anfordert und sich mit anderen transportierten Einheiten koordiniert. In allen oben zitierten Fällen ist diese vom Transportgut ausgehende Steuerung der Anlage jedoch nicht in Reinform, d. h. am Transportgut, umgesetzt worden. Es handelt sich hingegen meist um Stellvertreterprozesse in einem oder mehreren stationären Materialflussrechnern, die sozusagen im Auftrag des Transportguts handeln, d. h. als Makler bzw. Agenten. Die Intelligenz am Transportgut beschränkt sich auf einen RFID-Tag, der als dezentraler Datenspeicher oder auch nur als Identifikationshilfsmittel dient.

Die Gründe für den Stellvertreteransatz sind auf technische Herausforderungen und betriebswirtschaftliche Überlegungen zurückzuführen. $\mathrm{Zu}$ den technischen Herausforderungen zählen vor allem die Energieversorgung des mobilen Transportguts sowie die Anforderungen an die Leistung der elektronischen Komponenten (Intelligenz). Beispielsweise seien hier aktive Funkverbindungen genannt, die im Dauerempfang, durch Einsatz von Empfangsverstärkern, ein hohes Energieprofil vorweisen. Grundsätzlich bedeutet eine höhere Intelligenz des Transportguts meist auch einen Mehreinsatz von elektronischen Bauteilen (Sensoren, Aktoren, Displays etc.) und dementsprechend einen tendenziell höheren Energieverbrauch.

$\mathrm{Zu}$ den betriebswirtschaftlichen Überlegungen gehört die Abwägung der Mehrkosten beim Einsatz intelligenten Transportguts gegenüber den möglichen Einsparungen im logistischen Prozess, gerade auch im Vergleich zu existierenden logistischen Systemen. Dieser Abwägungsprozess ist durch die schnelle Entwicklung im Bereich der elektronischen Komponenten geprägt, so dass hier fortlaufend neue Resultate entstehen, sobald neue Komponenten auf den Markt kommen. Zusätzlich muss entschieden werden, ob und wie das Transportgut die logistische Intelligenz bereitstellt. Hierbei ist zwischen dem eigentlichen logistischen Objekt und dem Ladehilfsmittel zu unterscheiden. 
Diese Trennung ermöglicht eine Abstraktion vom eigentlichen Transportgut hin zur mobilen logistischen Einheit, die wiederverwendbar ist. Ein intelligentes Ladehilfsmittel steht dabei immer in Konkurrenz zu bisher existierenden, einfachen Ladehilfsmitteln: In der Regel darf ein intelligentes Ladehilfsmittel nicht viel kosten.

\section{DAs Projekt ViTol}

In diesem Abschnitt soll ein Forschungsprojekt vorgestellt werden, in dessen Rahmen bereits ein autonomes und intelligentes Transportgut entwickelt wurde. Dabei liegt der Fokus dieser Betrachtung auf den logistischen Funktionen, den Grad der logistischen Intelligenz, auf der Energieversorgung und den möglichen Kosten.

VitOL war ein dreijähriges Forschungsprojekt, das in den Jahren 2006 bis 2009 durchgeführt wurde (vgl. [HPS08]). Es führte die Idee des ,Internet der ge" konsequent fort, indem logistische Objekte wie Behälter und Paletten mit Sensorknoten ausgestattet wurden. Auf Basis dieser intelligenten Objekte wurden sowohl autonome Entscheidungen des Einzelobjektes als auch das kooperative Vorgehen eines Objektverbundes möglich.

Ziel waren die Entwicklung von Sensorknoten mit geeigneten Kommunikationsprotokollen, die Entwicklung und Umsetzung von Konzepten der Selbstorganisation, insbesondere des Gruppenmanagements, und die Implementierung der zugehörigen, auf den Sensorknoten ablauffähigen Applikationslogik. Die Demonstratoren Warensicherung, Materialflusssteuerung und Kommissionierung bildeten mögliche Einsatzgebiete von Sensornetzen in der Logistik ab.

Im Bereich der Warensicherung wurden Ladungsträger und Behälter mit Sensorknoten versehen. Ziel war die Überwachung der Behälter sowie die Kontrolle der Vollständigkeit einer zusammengestellten Menge von Behältern (eines Gebindes) entlang der Lieferkette.

Im Bereich der Materialflusssteuerung wurden Behälter und Steuerungskomponenten von Transportsystemen mit Sensorknoten ausgestattet. Materialbewegungen wurden durch den Einsatz der Sensorknoten dezentral initiiert und koordiniert und Ressourcenkonflikte lokal ausgehandelt. Die Aufgaben Steuerung und Transport innerhalb der Anlage wurden auf die Behälter und die Steuerungskomponenten verteilt.

Beim Demonstrator Kommissionierung wurde die Integration von RFID- und Sensorknoten-Technologie verfolgt, indem RFID-Reader und -Antennen an einen behälterlokalen Sensorknoten angeschlossen wurden. Bei der Kommissionierung kann so der Behälterinhalt des Entnahme- und des Kundenbehälters ermittelt und gegen den Kommissionierauftrag abgeglichen werden.
Die Testanlage des Fraunhofer IML zeichnete sich durch aktive Komponenten aus, die entsprechend ihrer Aufgaben und Systemsichten mit den notwendigen Daten und der Logik ausgestattet waren. Sie gestatteten damit den Anlagenbetrieb und Behältertransport. Allen Komponenten war es möglich, jederzeit und unabhängig von stationären Einrichtungen miteinander zu kommunizieren. So konnten Behälterinformationen unabhängig von Scannern oder RFID-Lesern abgefragt werden. Die aktiven Behälter lieferten Behälter- oder Umgebungsdaten, melden Stausituationen und konnten den eigenen Transportbedarf kommunizieren.

Der praktische Einsatz von Sensorknoten des ViTolProjekts wurde allerdings durch nicht gelöste Probleme eingeschränkt. $\mathrm{Zu}$ diesen gehören die Energiereichweite der verwendeten Behälterknoten und die hohen Anschaffungskosten für die Sensorknoten (vgl. [HPS08] im Fazit).

\section{DER INTELLIGENTE BEHÄLTER (INBIN)}

Aufbauend auf den Forschungsergebnissen des ViTol-Projekts soll in diesem Beitrag die Entwicklung eines intelligenten Behälters vorgestellt werden, der seine Energieversorgung vollständig durch Energy-Harvesting sicherstellt. In den folgenden Abschnitten werden zuerst die logistischen Funktionen des Behälters vorgestellt. Anschließend wird auf Energy-Harvesting im Allgemeinen und auf die Besonderheiten des Solar-Harvesting im logistischen Kontext eingegangen.

Analog zu den Behältern des ViTol-Projekts kennt und verwaltet der intelligente Behälter sowohl den eigentlichen Prozessablauf wie auch die dafür benötigten Prozessinformationen selbst. Dies ermöglicht eine im Aufbau einheitliche Steuerungseinheit, die jedoch flexibel und erweiterbar auf zahlreiche logistische Szenarien angepasst werden kann (siehe Abbildungen Abbildung 1 \& Abbildung 2).

Starre und vor allem ortsfeste Kommissioniersysteme werden durch Intelligente Behälter ersetzt. Dabei initiiert, kontrolliert und leitet der Behälter selbstständig den gesamten Kommissioniervorgang ein. Dies geschieht durch direkte Interaktion mit dem Kommissionierer, beispielsweise über das Display oder über ein „Pick-by-Voice“. System per Funk. Der gesamte Kommissioniervorgang (samt Bestätigungen und Fehlermeldungen) erfolgt so direkt am Behälter. Weitere Möglichkeiten eröffnen sich durch die Kombination des Intelligenten Behälters mit weiteren dezentralen Systemen, beispielsweise mit den Zellularen Transportsystemen. Durch dieses Zusammenspiel kann eine ortsvariable Kommissionierung umgesetzt werden. Kommissionierbereiche werden durch die autonomen Transportfahrzeuge spontan gebildet und auch wieder aufgelöst. 


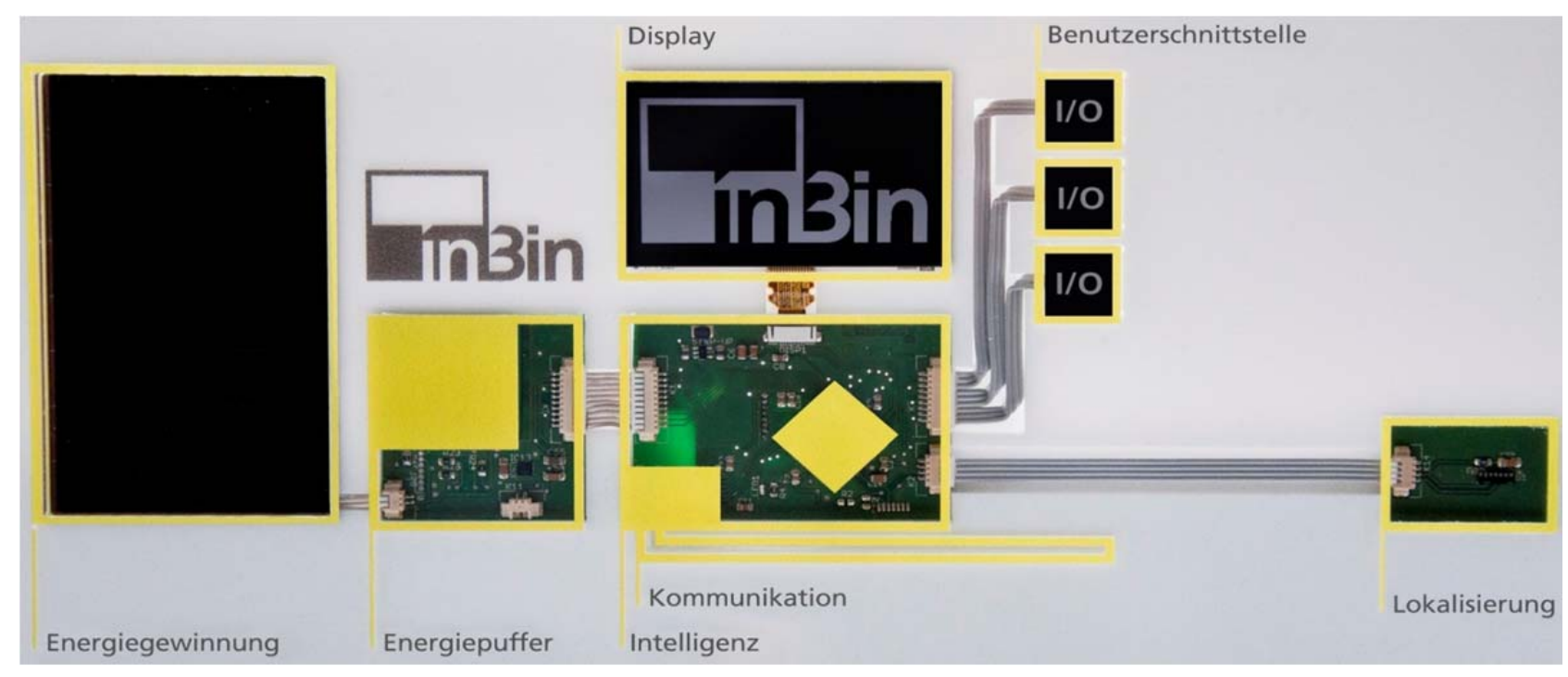

Abbildung 1. Aufbau der elektronischen Komponenten des intelligenten Behälters (inBin). Gut zu erkennen ist die Solarzelle zur Energiegewinnung auf der linken Seite. Über ein Display und Sensortasten ist eine einfache Mensch-MaschineKommunikation möglich.

Der Intelligente Behälter eignet sich als aktiver Teilnehmer für alle dezentral gesteuerten Materialflusssysteme nach dem Prinzip des Internet der Dinge. Eine zentrale Materialflusssteuerung kann durch die Verteilung der Steuerungsintelligenz auf die Förderelemente und die Behälter entfallen. Weiterhin ist eine Kombination mit den Zellularen Transportsystemen einfach möglich, da die Intelligenten Behälter die Transportfahrzeuge aktiv anfordern und für logistische Aufgaben nutzen können, zum Beispiel für den Transport oder die Sequenzbildung.

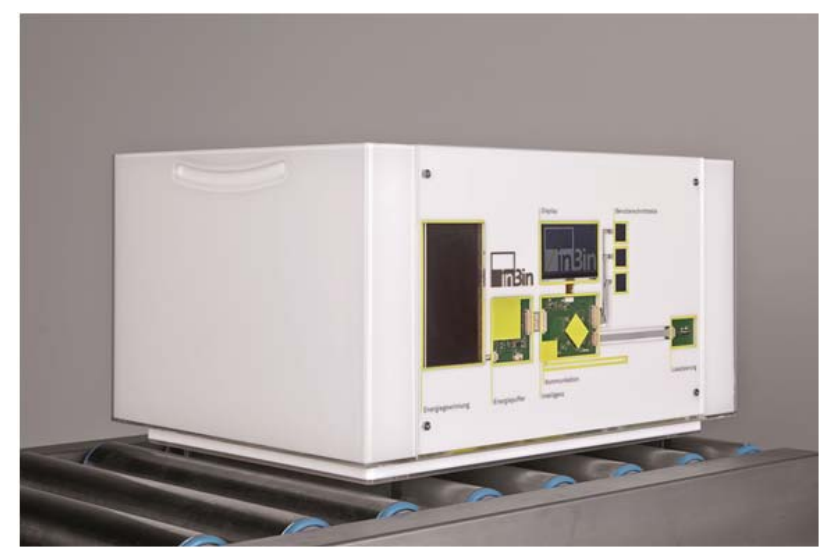

Abbildung 2. Der inBin in einer Demonstrationsvariante. Die Elektronik lässt sich mit geringem Aufwand in Standardbehälter integrieren.

Jeder Behälter hat eine eindeutige Identifikationsnummer, über die er weltweit identifizierbar und nachverfolgbar ist. Die Identifikationsnummer kann über verschiedene Schnittstellen, die der Behälter bietet, abgefragt und kontrolliert werden. Zusätzlich kann der Intelligente Behälter durch den Einsatz von Sensorik seine Umge- bungsparameter erfassen und so beispielsweise eine Temperaturüberwachung gewährleisten.

Der modulare Aufbau des Intelligenten Behälters erlaubt eine individuelle Anpassung an fast alle logistischen Prozesse:

- Schnittstelle: Die Kommunikation zum Menschen kann entweder direkt über ein Display (beispielsweise LCD oder e-Ink) und Knöpfe erfolgen oder der Behälter bietet Schnittstellen zu externen Systemen, beispielweise „Pick-byLight" - oder „Pick-by-Voice“-Lösungen.

- Energiequelle: Der Einsatz von Energy-Harvestern macht den Einsatz unter verschiedensten Umgebungsbedingungen möglich. Die Energie kann beispielsweise über Licht (Solar) oder Erschütterungen (Vibration) erzeugt werden.

- Energiepuffer: Durch die Kombination mit verschiedenen Zwischenspeichern (Energiepuffern) kann die Dunkellaufzeit des intelligenten Behälters individuell angepasst werden.

- Intelligenz: Variable Speichergrößen und Rechenkapazitäten erlauben stufenweise eine Anpassung der „Intelligenz“ des Behälters an den gewünschten Grad der Dezentralität.

- Kommunikation: Es können beliebige (proprietäre / offen-standardisierte) Kommunikationsprotokolle (beispielsweise 6LoWPAN oder ZigBeePro) eingesetzt werden. Dies ermöglicht die nahtlose Integration in fast jede bestehende Kommunikationsinfrastruktur im Unternehmen. 
Grundsätzlich gilt die Regel, dass mehr Funktionen mehr Bauteile benötigen, die wiederum den Energieverbrauch erhöhen. Daher sollen im folgenden Abschnitt Energiequellen betrachtet werden, die für die Verwendung in der Intralogistik in Betracht kommen.

\section{ENERGIEQUELLEN FÜR INTELLIGENTE SYSTEME IN DER INTRALOGISTIK}

Für die Realisierung des inBin wurde die gesamte Energieversorgung aller Komponenten durch EnergyHarvesting umgesetzt. Dies verursacht auf den ersten Blick Mehrkosten (im diesem Fall von etwa $8 €$ Materialkosten), die sich allerdings bei der Betrachtung der gesamten Laufzeit relativieren und sogar als weitaus beste Variante herausstellen. Daher wurden verschiedene Möglichkeiten der Energieversorgung von intelligenten Ladungsträgern betrachtet. Dabei wird eine Laufzeit von etwa 10 Jahren ebenso berücksichtigt wie auch die Baugröße, die Wartungsfreundlichkeit, der Schutz vor Umwelteinflüssen und die Umweltverträglichkeit.

Primärbatterien, welche die gesamte Kapazität für eine Laufzeit von 10 Jahren vorhalten, scheiden aus mehreren Gründen aus. Unter Einbeziehung der Selbstentladung müsste eine solche Primärbatterie mindestens $8.000 \mathrm{mAh}$ Kapazität haben. Lithium-Thionylchlorid-Batterien, welche die Anforderungen an Haltbarkeit und Kapazität erfüllen, sind im Vergleich zu den restlichen Komponenten teuer und haben ein größeres Volumen als alle Komponenten des inBin zusammen.

Eine Alternative auf dem ersten Blick sind wechselbare Primärbatterien (i. d. R. Knopfzellen). Diese sind klein, günstig und haben eine Kapazität für etwa $1 \mathrm{Jahr}$ Betriebsbereitschaft. Allerdings ergeben sich durch den notwendigen Batteriewechsel andere Probleme:

- Jeder Wechsel verursacht Wartungskosten.

- Die Elektronik kann nicht hermetisch abgeriegelt werden, da ein Vergießen nicht möglich ist. Ein optimaler Schutz gegen Umwelteinflüsse ist nicht möglich.

- $\quad$ Ein Wechsel der Batterie erfordert in der Regel auch einen Austausch von Dichtungen.

- Eine ständige Kontrolle der Batterieladung ist erforderlich, um eine rechtzeitige Ausschleusung der Ladungsträger zu ermöglichen.

Gemeinsam haben alle Primärbatterien das Entsorgungsproblem durch ihre umweltschädlichen Bestandteile sowie Sicherheitsrisiken durch z. B. Explosion und Selbstentzündung (Lithiumbatterien sind Gefahrgut).

Über die gesamte Laufzeit betrachtet ergeben sich deutliche Kostenvorteile bei Anwendung von EnergyHarvesting. Beim Vergleich werden Wartungskosten von
$5 €$ pro Batteriewechsel angenommen. Nach 3 Jahren Betrieb ergeben sich Kostenvorteile beim Energy-Harvesting gegenüber den Primärbatterien (siehe Tabelle 1).

Tabelle 1. $\quad$ Übersicht über die Kostenfaktoren beim Einsatz von Batterien im Gegensatz zu Energy-Harvesting

\begin{tabular}{|l|l|l|l|l|}
\hline & $\begin{array}{l}1 . \\
\text { Jahr }\end{array}$ & $\begin{array}{l}3 . \\
\text { Jahr }\end{array}$ & $\begin{array}{l}5 . \\
\text { Jahr }\end{array}$ & $\begin{array}{l}10 . \\
\text { Jahr }\end{array}$ \\
\hline $\begin{array}{l}\text { Materialkosten für } \\
\text { Energy-Harvesting } \\
\text { (in €) }\end{array}$ & 8 & 8 & 8 & $\mathbf{8}$ \\
\hline $\begin{array}{l}\text { Materialkosten für } \\
\text { Primärbatterie } \\
\text { (in €) }\end{array}$ & 0,60 & 1,80 & 3 & 6 \\
\hline $\begin{array}{l}\text { Wartungskosten } \\
\text { Primärbatterie } \\
\text { (in €) }\end{array}$ & 0 & 10 & 20 & 45 \\
\hline $\begin{array}{l}\text { Gesamtkosten } \\
\text { Primärbatterie } \\
\text { (in €) }\end{array}$ & 1,20 & 11,80 & 23 & $\mathbf{5 1 €}$ \\
\hline
\end{tabular}

\section{ENERGY-HARVESTING IN DER INTRALOGISTIK}

Zurzeit gibt es vier generelle Methoden zur Energiegewinnung (vgl. [MW12]) aus der Umwelt, wobei deren Eigenschaften sehr unterschiedlich sind und sich nur eine für das Einsatzfeld des inBin eignet.

\subsection{Photovoltaische WANDLER}

Die Wirkungsweise der Solarzellen basiert auf dem Photoeffekt. Durch die Zufuhr von Strahlungsenergie werden Ladungsträger freigesetzt und eine Gleichspannung erzeugt. Der Photoeffekt ist die am besten erforschte Variante des Energy-Harvesting mit einem guten Wirkungsgrad und im Vergleich geringen Kosten.

\subsection{THERMOELEKTRISCHE WANDLER}

Thermoelektrische Wandler setzen thermische Unterschiede durch Nutzung des Seebeckeffekts in elektrische Energie um. Thermoelektrische Wandler sind im Vergleich zu ihrer Leistung sehr klein, benötigen aber zum Betrieb eine deutliche Temperaturdifferenz. Dies erfordert häufig den Einsatz eines Kühlkörpers auf der kälteren Seite des Thermowandlers.

\subsection{BEWEGUNGS- UND VIBRATIONSWANDLER}

Bewegungs- und Vibrationswandler arbeiten im niederfrequenten Ultraschallbereich zur Energiegewinnung. Sie können als Resonanz- oder Impulswandler arbeiten und dabei gleichzeitig als Sensor zur Datengewinnung (Schock- oder Vibrationssensor) eingesetzt werden. Durch Nutzung der Mikrosystemtechnik sind sehr kompakte Bauformen möglich. 


\subsection{RF-WANDLER}

Radio-Frequency-Wandler konvertieren elektro-magnetische Funkwellen in elektrische Energie. Die Energie steht als Wechselspannung zur Verfügung und muss zur Gleichspannungsnutzung zunächst gleichgerichtet werden. Die Energiegewinnung aus bereits in der Umwelt vorhandenen Funkwellen befindet sich noch im Forschungsstadium und hat weist zurzeit nur sehr geringe Energieerträge auf. Anders sieht es aus bei Verwendung von aktiven Erregerantennen, hierbei handelt es sich jedoch nicht mehr um Energy-Harvesting aus der Umgebung sondern um eine Form der aktiven Energieübertragung. Diese Technik wird in der Logistik intensiv bei RFID-Systemen eingesetzt. Tabelle 2 gibt eine Übersicht über die Größenordnungen von Leistungsdichten und Wirkungsgraden bei Einsatz von Energy-Harvesting.

Tabelle 2. $\quad$ Übersicht über die Größenordnungen der Leistungsdichte und Wirkungsgrade verschiedener Energy-Harvesting-Methoden

\begin{tabular}{|l|l|l|l|}
\hline \multirow{2}{*}{ Wandler } & Umfeld & $\begin{array}{l}\text { Leistungs- } \\
\text { dichte }\end{array}$ & $\begin{array}{l}\text { Wir- } \\
\text { kungs- } \\
\text { grad }\end{array}$ \\
\hline \multirow{2}{*}{ Photovoltaik } & $\begin{array}{l}\text { Außen- } \\
\text { bereich }\end{array}$ & $\begin{array}{l}100 \\
\mathrm{~mW} / \mathrm{cm}^{2}\end{array}$ & \multirow{2}{*}{$10-24 \%$} \\
\cline { 2 - 3 } & $\begin{array}{l}\text { Innen- } \\
\text { bereich }\end{array}$ & $0,1 \mathrm{~mW} / \mathrm{cm}^{2}$ & \\
\hline $\begin{array}{l}\text { Thermo- } \\
\text { elektrisch }\end{array}$ & Industrie & $\begin{array}{l}1-10 \\
\mathrm{~mW} / \mathrm{cm}^{2}\end{array}$ & \multirow{2}{*}{$1-3 \%$} \\
\hline \multirow{2}{*}{$\begin{array}{l}\text { Vibrations- } \\
\text { wandler }\end{array}$} & $\begin{array}{l}\text { Hz- } \\
\text { Bereich }\end{array}$ & $\begin{array}{l}0,004 \\
\mathrm{~mW} / \mathrm{cm}^{3}\end{array}$ & \multirow{2}{*}{$25-50 \%$} \\
\cline { 2 - 3 } & $\begin{array}{l}\mathrm{kHz}- \\
\text { Bereich }\end{array}$ & $0,8 \mathrm{~mW} / \mathrm{cm}^{3}$ & \\
\hline \multirow{2}{*}{ RF-Wandler } & Sub-gHz & $\begin{array}{l}0,0001 \\
\mathrm{~mW} / \mathrm{cm}^{2}\end{array}$ & \multirow{2}{*}{$50 \%$} \\
\cline { 2 - 3 } & WiFi & $\begin{array}{l}0,000001 \\
\mathrm{~mW} / \mathrm{cm}^{2}\end{array}$ & \multicolumn{2}{|c}{} \\
\hline
\end{tabular}

\section{SOLAR-HARVESTING IN DER INTRALOGISTIK}

Nur Licht und kinetische Energie erweisen sich nach [KKH11] als verwertbar (siehe Abbildung 3). Für die sinnvolle Aufnahme der kinetischen Energie sind ein regelmäßiges Auftreten von Vibration und Stoßbewegungen notwendig. Dies trifft nur auf Ladungsträger zu, die einen Großteil ihrer Verwendungszeit auf Stetigfördertechnik bewegt werden. Da die Funktionsfähigkeit der intelligenten Ladungsträger aber auch z. B. im Lager gewährleistet werden soll, scheidet die kinetische Energie als potentielle Energiequelle aus.

Eine Besonderheit gegenüber typischen EnergyHarvesting-Anwendungen sind die extremen Lichtverhältnisse der typischen Einsatzumgebung der intelligenten Ladungsträger. Um die Problematik des sogenannten „Indoor-Energy-Harvesting“ zu erläutern, ist eine Betrach- tung der auf die Solarzellen einwirkenden Energie in Innenräumen notwendig (vgl. [CO12]).

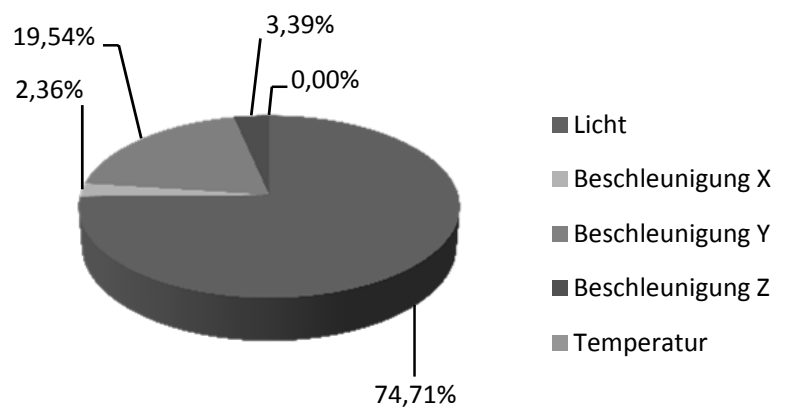

Abbildung 3. Übersicht über die potentiellen Energiequellen in intralogistischen Anlagen [KKH11]

Im Verhältnis zu gängigen Solaranwendungen unter Ausnutzung der direkten Sonneneinstrahlung ist die zu erwartende Strahlungsenergie im Innenbereich um ein Vielfaches kleiner. Bei direkter Sonneneinstrahlung liegt die Strahlungsenergie in der Größenordnung von mindestens $1.000 \mathrm{~W} / \mathrm{m}^{2}$ auf der Solarzellenoberfläche. Im Innenbereich wird dagegen von etwa $1 \mathrm{~W} / \mathrm{m}^{2}$ ausgegangen. Die folgende Rechnung zeigt, dass im Innenbereich typischer Logistikgebäude noch wesentlich weniger Licht zur Verfügung stehen kann:

Die Energiedichte einer idealisierten $100 \mathrm{~W}$ Glühbirne in einem Abstand von $1,5 \mathrm{~m}$ zur Solarfläche beträgt $3,53 \mathrm{~W} / \mathrm{m}^{2}$ :

$$
\begin{gathered}
\text { Energiedichte }_{\text {Glühbirne }}=\frac{100 \mathrm{~W}}{4 \pi \mathrm{r}^{2}}=3,53 \mathrm{~W} / \mathrm{m}^{2}, \\
(\text { mit } \mathrm{r}=1,5 \mathrm{~m})
\end{gathered}
$$

Im industriellen Umfeld sind Glühbirnen fast vollständig durch Fluoreszenzlampen ersetzt worden. Fluoreszenzlampen erzeugen bei gleicher Leistung einer Glühbirne einen für das menschliche Auge vierfach größeren Helligkeitseindruck. Dies liegt daran, dass Fluoreszenzlampen mit ihrem Farbspektrum (siehe Abbildung 4, Fluorescent light) besonders gut zur maximalen Farbempfindlichkeit des Auges bei $555 \mathrm{~nm}$ Wellenlänge passen.

Aufgrund dieser spezifischen Eigenschaft des Auges wird Helligkeit als Strahlungsenergie pro Fläche bei einer Wellenlänge von $555 \mathrm{~nm}$ definiert und mit der Einheit $1 \mathrm{x}$ (,Lux“) benannt.

$$
1 \mathrm{~lx}=1 \text { lumen } / \mathrm{m}^{2}
$$

1 lumen $=\frac{1}{683} \mathrm{~W}$, bei $555 \mathrm{~nm}$ 


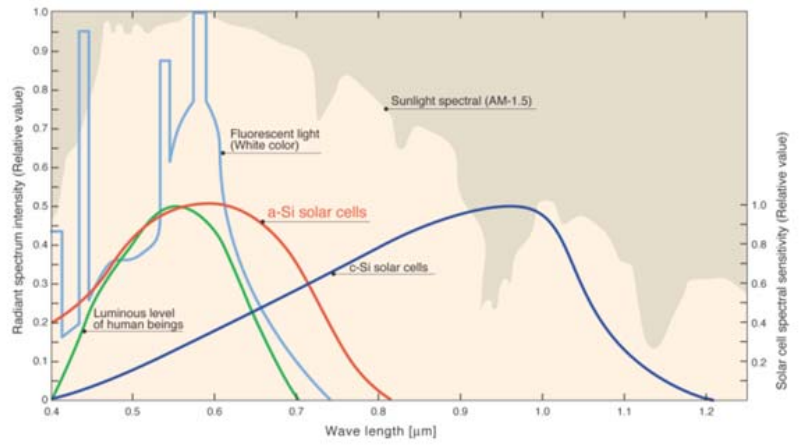

Abbildung 4. Spektrum des Sonnenlichts und das einer Fluoreszenzlampe. Im Vergleich dazu die Empfindlichkeit des menschlichen Auges, einer amorphen Indoor-Solarzelle und einer kristallinen Outdoor-Solarzelle [Tom04]

Aus diesem Grund hat eine Fluoreszenzlampe mit der gleichen Helligkeit einer $100 \mathrm{~W}$ Glühbirne nur eine Energiedichte von $0,88 \mathrm{~W} / \mathrm{m}^{2}$ bei gleichem Abstand.

$$
\begin{gathered}
\text { Energiedichte }_{\text {Fluoreszenzlampe }}= \\
\frac{25 \mathrm{~W}}{4 \pi \mathrm{r}^{2}}=0,88 \mathrm{~W} / \mathrm{m}^{2},(\text { mit } \mathrm{r}=1,5 \mathrm{~m})
\end{gathered}
$$

Daher sind solargetriebene Indoor-Energy-Harvesting-Anwendungen nicht vergleichbar mit denen, die mit Sonnenlicht betrieben werden. Selbst bei gleicher Helligkeit hat das Licht einer Hallenbeleuchtung nur einen Bruchteil der Energie des Sonnenlichts. Hinzu kommt, dass beim Wirkungsgrad für Outdoor-Solarzellen (siehe Abbildung 4, c-Si solar cells) heutzutage Wirkungsgrade von über $20 \%$ erreicht werden, während bei IndoorSolarzellen (siehe Abbildung 4, a-Si solar cells) unter Kunstbeleuchtung nur etwa 6\%-10\% erreicht werden (vgl. [Tom04]).

Zur Bestimmung der Machbarkeit des inBin wurden zwei typische Logistikhallen auf ihre Helligkeit an typischen Behälterstandorten untersucht. Um einen relativen Vergleich für diese Helligkeitswerte $\mathrm{zu}$ erhalten, sind Vergleichswerte natürlicher Beleuchtungen und empfohlene Beleuchtungsstärken für Arbeitsplätze (DIN5038) in Tabelle 3 aufgenommen worden.

Es wird daher angenommen, dass am Ort der Kommissionierung, an dem auch der größte Energiebedarf besteht (Display, Interaktion, Sensortaster), eine Helligkeit von 250 - 500 lx herrscht. Bei einem eingelagerten Behälter wird noch eine Resthelligkeit von 20 lx angenommen.

Um die notwendige Solarfläche und damit die Machbarkeit zu ermitteln, muss der mittlere Energiebedarf $\mathrm{P}_{\text {Durchschnitt }}$ aller Verbraucher bekannt sein. Im Pilotprojekt inBin wurden dafür 12 Komponenten ermittelt, die für den hauptsächlichen Energiebedarf verantwortlich sind. Jede Komponente $k$ kann je nach Funktionsumfang unterschiedliche Energieverbräuche haben. Jeder dieser $\mathrm{Zu}$ - stände $z$ hat, bei einem vorher festgelegten Szenario (z. B. alle 5 Sekunden 20 Byte senden und 50 Byte empfangen), einen statistischen Zeitanteil $t_{i, j}$ an der Gesamtzeit.

Tabelle 3. $\quad$ Übersicht über die Lichtstärken in verschiedenen intralogistischen und natürlichen Umgebungen

\begin{tabular}{|l|c|}
\hline Umgebung & Lichtstärke in lx \\
\hline $\begin{array}{l}\text { Lagerhalle (mit Oberlichtern), } \\
\text { zwischen Hochregalen }\end{array}$ & $50-150$ \\
\hline $\begin{array}{l}\text { Lagerhalle (mit Oberlichtern), } \\
\text { auf der Fördertechnik }\end{array}$ & $150-600$ \\
\hline $\begin{array}{l}\text { Lagerhalle (ohne Oberlichter), } \\
\text { auf der Fördertechnik }\end{array}$ & $80-250$ \\
\hline $\begin{array}{l}\text { Lagerhalle (ohne Oberlichter), } \\
\text { zwischen Hochregalen }\end{array}$ & $500-1.000$ \\
\hline $\begin{array}{l}\text { Arbeitsplatzbeleuchtung Büro } \\
\text { (DIN5035) }\end{array}$ & $250-500$ \\
\hline $\begin{array}{l}\text { Arbeitsplatzbeleuchtung Schlos- } \\
\text { ser (DIN5035) }\end{array}$ & 1 \\
\hline Mondlicht & 10.000 \\
\hline Sonnenlicht (im Schatten) & 100.000 \\
\hline Sonnenlicht (direkt) & \\
\hline
\end{tabular}

Der durchschnittliche Energiebedarf aller Komponenten lässt sich dann wie folgt ermitteln (vgl. [CO12]):

$$
P_{\text {Durchschnitt }}=\sum_{i=1}^{K} \sum_{j=1}^{Z} V_{i} \times I_{i, j} \times T_{i, j}
$$

K: Menge der Komponenten

\section{Z: Menge der Zustände der einzelnen Komponenten}

$v_{i}$ : Die Versorgungsspannung der Komponente $i$. Innerhalb eines Systems können mehrere Versorgungsspannungen verwendet werden, um die Verlustleistung der einzelnen Komponenten zu reduzieren.

$i_{i, j}$ : Strombedarf der Komponente $i$ im Zustand $j$

$t_{i, j}$ : Der Zeitanteil des Zustands $j$.

Die Herausforderung bei der Entwicklung der Elektronik ist, $P_{\text {Durchschnitt }}$ geringer zu halten als den durchschnittlichen Ertrag der Solarzellen. Das vorherige Beispiel der Energiesparlampe aufgreifend ergibt sich eine Energieausbeute pro Quadratzentimeter Solarfläche von $0,0088 \mathrm{~mW}$. Eine normale LED hat im Vergleich dazu einen Energiebedarf von $40 \mathrm{~mW}$.

$$
\frac{0,88 \mathrm{~W} / \mathrm{m}^{2}}{10000} \cdot \eta=0,0088 \mathrm{~mW} / \mathrm{cm}^{2}, \text { mit } \eta=0,1
$$

Für die Realisierung des inBin wurde eine effektive Solarfläche von ca. $50 \mathrm{~cm}^{2}$ gewählt. In ersten Versuchen wurde damit eine Energieausbeute von mindestens $0,05 \mathrm{~mW}$ und im Durchschnitt $0,2 \mathrm{~mW}$ erzielt. Mit dieser 
Energie können alle Komponenten des inBin dauerhaft betrieben werden.

\section{$7 \quad$ FAZIT}

Intelligente und vollständig energieautarke Ladungsträger sind durch die Entwicklung neuer sparsamer, elektronischer Komponenten technisch realisierbar geworden. Der inBin verdeutlicht den Trend hin zu einem tatsächlichen Internet der Dinge, in dem die Intelligenz mobil das Transportgut begleitet. Eine Herausforderung für die weitere Forschung besteht in der Entwicklung großer Systeme mit einer Vielzahl von intelligenten Ladehilfsmitteln.

\section{LITERATUR}

[BH07] Bullinger, Hans-Jörg; ten Hompel, M. (Hrsg.): Internet der Dinge. Berlin, Heidelberg, New York: Springer, 2007

[CO12] Chevvrier, Matthieu; Odenwald, Thomas: Technische Herausforderung und deren Lösungen bei dem Entwurf von Indoor Photovoltaik-Produkten. In: Elektronik energy harvesting congress 2012, 2012

[DIN5035] DIN Deutsches Institut für Normung e. V: Beleuchtung mit künstlichem Licht- Teil 8: Arbeitsplatzleuchten Anforderungen, Empfehlungen und Prüfung. Norm, 2007

[GCK08a] Günthner, Willibald A.; Chisu, Razvan; Kuzmany, Florian: Das "Internet der Dinge" - Teil I: Intelligent verteilt. In: $\mathrm{F}+\mathrm{H},(7-8)$, S. 422-425, 2008

[GCK08b] Günthner, Willibald A.; Chisu, Razvan; Kuzmany, Florian: Das "Internet der Dinge" - Teil II: Steuern ohne Hierarchie. In: F+H, (9), S. 494-497, 2008

[HFR08] ten Hompel, Michael; Follert, Guido; Roidl, Moritz: Künstliche Intelligenz im Internet der Dinge: Die Zukunft der Materialflusssteuerung mit autonomen Agenten. In: Wolf-Kluthausen, Hanne (Hrsg.): Jahrbuch Logistik 2008. S. 2429. Korschenbroich: free Beratung $\mathrm{GmbH}, 2008$

[HPS08] ten Hompel, Michael; Pöter, Elisabeth; Schier, Arkadius: Dezentrale Materialflusssteuerung unter Einsatz von drahtlosen Sensornetzwerken. In: Tagungsband zum 4. Fachkolloquium der
Wissenschaftlichen Gesellschaft für Technische Logistik e. V., Chemnitz 2008

[KKH11] Kamagaew, Andreas; Kirks, Thomas; ten Hompel, Michael: Energy potential detection for autarkic Smart Object design in facility logistics. In: Control System, Computing and Engineering (ICCSCE), 2011 IEEE International Conference on, S. 285-290, 2011

[May09] Mayer, Stephan H..: Development of a completely decentralized control system for modular continuous conveyors. Karlsruhe: Dissertation, Universität Karlsruhe (TH), 2009

[MW12] Mackensen, Elke; Wendt, Thomas M.: Energy-Harvesting-basierte Energieversorgungen für drahtlose SensorSysteme: Analyse kommerziell verfügbarer Lösungen und daraus abgeleitete Design-Konzepte. In: Elektronik energy harvesting congress 2012, 2012

[NH10] Nopper, Jan Robert; ten Hompel, Michael: Zur Bewertung von Wandelbarkeit in der Intralogistik. In: Logistics Journal Proceedings. Online verfügbar unter

http://dx.doi.org/10.2195/LJ_PROC10_ Nopper. 2010

[Tom04] Tomohiro, Ohkubo: Sanyo Amorphous Solar Cells. SANYO Electric Co., Ltd Semiconductor Company, Tokyo, 2004 